\title{
Helicity of Solar Active Regions from a Dynamo Model
}

\author{
Piyali Chatterjee \\ Department of Physics, Indian Institute of Science, Bangalore 560 012, India. \\ e-mail: piyali@physics.iisc.ernet.in
}

\begin{abstract}
We calculate helicities of solar active regions based on the idea that poloidal flux lines get wrapped around a toroidal flux tube rising through the convection zone, thereby giving rise to the helicity. We use our solar dynamo model based on the Babcock-Leighton $\alpha$-effect to study how helicity varies with latitude and time.
\end{abstract}

Key words. MHD—Sun: activity—Sun: magnetic fields—sunspots.

\section{Introduction}

Typically, solar active regions are known to have helicity associated with them and the observational studies based on vector magnetograms (Seehafer (1990); Pevtsov et al. (1995, 2001); Abramenko et al. (1997) and Bao \& Zhang (1998)) indicate that the preferred sign of this helicity is opposite in the two hemispheres (negative in northern hemisphere and positive in the southern hemisphere), in spite of a very large statistical scatter. Figure 2 of Canfield \& Pevtsov (2000) is a typical plot showing a variation of helicity with latitude, which any theoretical model has to explain.

Solar magnetic fields are believed to be produced by the dynamo process. One possibility is that the dynamo process itself is responsible for the generation of helicity. The other possibility is that the rising flux tubes, which eventually form active regions, get the twist by interacting with the helical turbulence in the surrounding convection christened the $\Sigma$-effect by Longcope et al. (1998). The two possibilities mentioned above need not be mutually exclusive: both may be simultaneously operative. A careful comparison between observational data and detailed theoretical models will be needed to ascertain the relative importance of these two effects.

We present here calculations of helicity based on our two-dimensional kinematic solar dynamo model presented in Nandy \& Choudhuri (2002) and Chatterjee et al. (2004).

The dynamo equation deals with the mean magnetic field (see, for example, Choudhuri 1998, Ch. 16), whereas we want to find helicities of active regions which form from flux tubes. To make a connection between these two, we have to look at the relation between dynamo theory and flux tubes. In a Babcock-Leighton dynamo poloidal field $A$ is created from decay of tilted active regions, the amount of tilt being given by the Joy's Law. Also in accordance with the Hale's polarity rule, a bipolar active region formed by a flux tube with positive $B_{\phi}$ would have the leading spot towards the equator than the following spot. Decay of such a pair would thus mean clockwise lines of $B_{p}$ around active regions. When a new toroidal flux tube with positive $B_{\phi}$ moves upwards 
near the surface, the poloidal field gets wrapped around the toroidal flux tube. Due to high magnetic Reynolds number the flux tubes are not able to cut through the poloidal field lines thus giving rise to the helicity.

\section{Estimating the value of helicity}

For force free fields in the photosphere and the corona we may define helicity as

$$
\alpha=\frac{(\nabla \times \mathbf{B})_{z}}{B_{z}},
$$

where $z$ corresponds to the vertical direction, which is along the axis of the flux tube for active regions on the surface. The parameter $\alpha$ (not to be confused with the dynamo $\alpha$-effect traditionally associated with the poloidal field generation mechanism) is a measure of the handedness or chirality of the magnetic field. This twist parameter is an indicator of how stressed the active region flux system is and it is known to play an important role in the flaring and explosive activity of active region magnetic fields (Canfield et al. 1999; Nandy et al. 2003). The typical observed value of the twist parameter $\alpha$ from magnetoram data, calculated by several authors is about $2 \times$ $10^{-8} \mathrm{~m}^{-1}$.

To estimate the value of helicity theoretically, we have to keep in mind that the flux of poloidal field $B_{P}$ through the whole SCZ gets dragged by the toroidal flux tube rising under magnetic buoyancy (see Fig. 4 of Choudhuri 2003). If $d$ is the depth of the convection zone, the flux dragged by the tube is

$$
F \approx B_{P} d .
$$

This flux $F$ gets wrapped around the tube of radius $a$. In an ideal-MHD situation, this flux $F$ would be confined to a narrow sheath around the flux tube. In reality, however, we expect that the turbulence around the flux tube would make this flux $F$ penetrate into the flux tube. Then the magnetic field going around the tube can be taken to be of order $F / a$. The current density $|\nabla \times \mathbf{B}|$ associated with this field is of order $F / a^{2}$ and is along the axis of the tube. If $B_{T}$ is the magnetic field inside the flux tube, then it follows from (1):

$$
\alpha \approx \frac{F / a^{2}}{B_{T}} \approx \frac{B_{P} d}{B_{T} a^{2}}
$$

on substituting from (2) for $F$. We use $B_{P} \approx 1 \mathrm{G}$, the depth of the SCZ $d \approx 2 \times 10^{8} \mathrm{~m}$ and the field inside sunspots $B_{T} \approx 3000 \mathrm{G}$. On taking the radius of the sunspot $a \approx 2000 \mathrm{~km}$ and $a \approx 5000 \mathrm{~km}$, we get $\alpha \approx 2 \times 10^{-8} \mathrm{~m}^{-1}$ and $\alpha \approx 3 \times 10^{-9} \mathrm{~m}^{-1}$ respectively. Thus, from very simple arguments, we get the correct order of magnitude.

\section{Results from dynamo simulation}

In section 4 of Chatterjee et al. (2004) we have presented a particular dynamo model which we refer to as our standard model. We now present helicity calculations based on this standard model. 


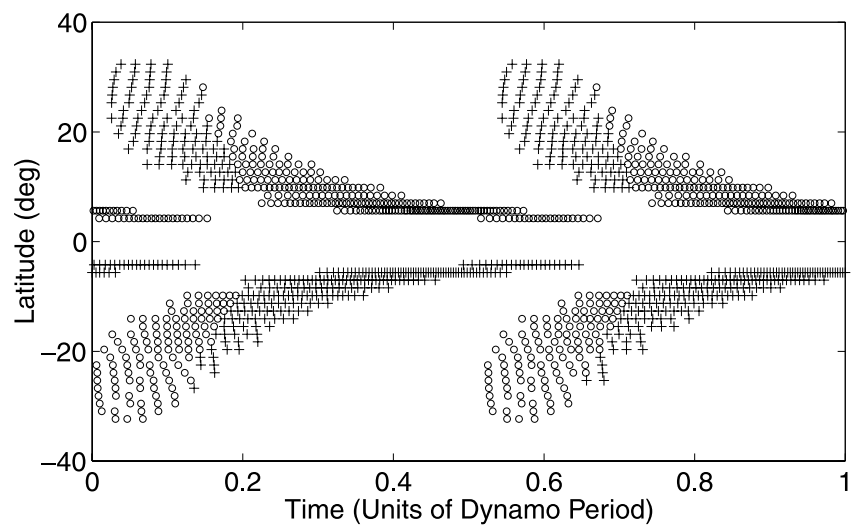

Figure 1. Theoretical butterfly diagram of eruptions from our standard dynamo model. Eruptions with positive and negative helicities are denoted by ' + ' and 'o' respectively.

A flux eruption takes place in our model whenever the toroidal field at the bottom of the SCZ exceeds a critical value. Whenever an eruption takes place in our dynamo simulation, we calculate the poloidal flux $F$ through SCZ at the eruption latitude by integrating $B_{\theta}$ from the bottom of $\operatorname{SCZ}\left(r=R_{b}\right)$ to the top $\left(r=R_{\odot}\right)$, i.e.,

$$
F=\int_{R_{b}}^{R_{\odot}} B_{\theta} d r .
$$

The value of $F$ calculated at the eruption latitude at the time of eruption gives the amplitude of helicity associated with the eruption. If the sign of $F$ is opposite to the sign of the toroidal field $B$ at the bottom of SCZ, then the helicity is taken as negative (otherwise it is positive). Figure 1 shows the simulated butterfly diagram, indicating active regions of positive and negative helicity. During the solar maximum, the helicity is negative in the northern hemisphere and positive in the southern, as we expect. However, at the beginning of a cycle, there is a short duration when the sign of helicity is 'wrong', i.e., opposite of the preferred helicity. We find that $67 \%$ of the eruptions in our simulation have 'correct' helicity.

Figure 2(a) is a plot of helicity associated with eruptions at different latitudes. This is the theoretical plot that has to be compared with observational plots like Fig. 2 of Canfield \& Pevtsov (2000). We notice that the theoretical plot has considerably less scatter compared to the observational data.

To see the variation of helicity with the cycle, Fig. 2(b) and Fig. 2(c) present plots of helicity for eruptions during 4 years of solar maximum and 4 years at the beginning of the cycle respectively. The straight lines represent the least-square fits. For 'correct' helicity (negative in north and positive in south), the gradient $d \alpha / d \lambda$ of the straight line has to be negative, as we see in Fig. 2(b) corresponding to solar maximum ( $\lambda$ is the latitude). The gradient, however, is positive at the start of the cycle. To find out how this gradient varies with the cycle, we divide the cycle period into 16 equal intervals and then find the gradient $d \alpha / d \lambda$ for each of the intervals by using eruptions during that interval. Figure 3 shows how the gradient $d \alpha / d \lambda$ varies with the solar cycle. If the $\Sigma$-effect makes a significant contribution in the production of helicity, then the variation with the cycle may be less pronounced compared to what we find in our model without 

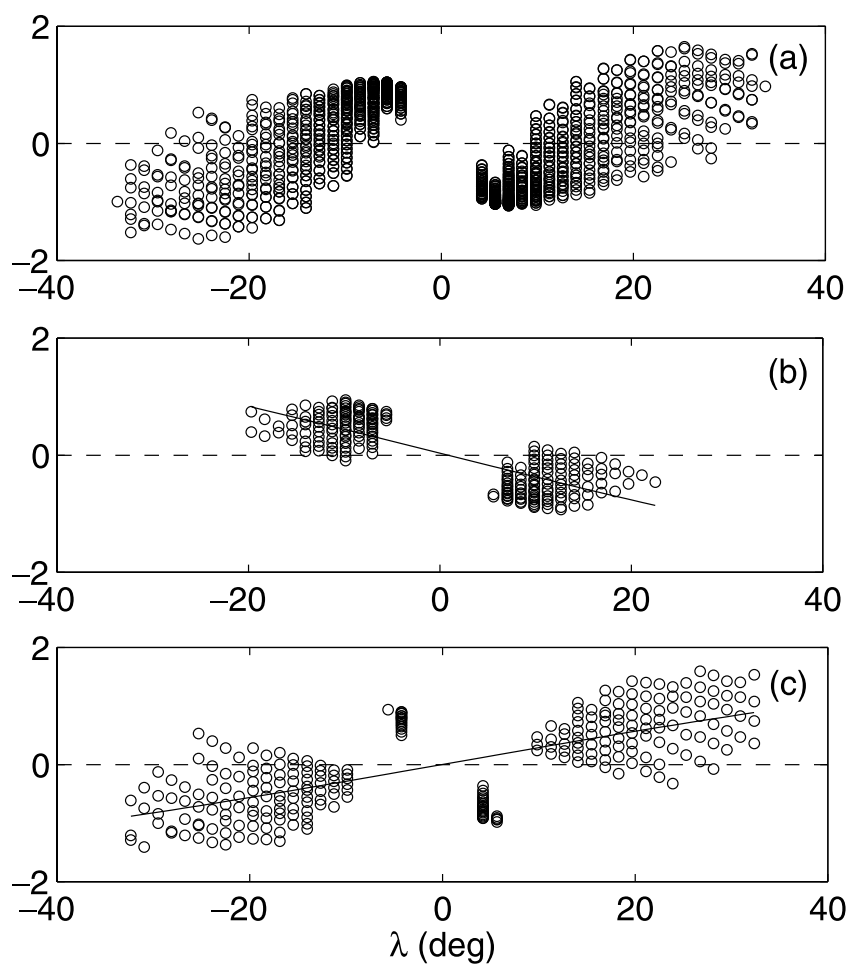

Figure 2. Helicity $\alpha$ (plotted along the vertical axis in arbitrary units) for eruptions at different latitudes, denoted by open circles: (a) for an entire solar cycle; (b) for 4 years during the maximum and the declining phases of the solar cycle; (c) for the first 4 years of the solar cycle. The solid lines in (b) and (c) are least-square fits to the model results.

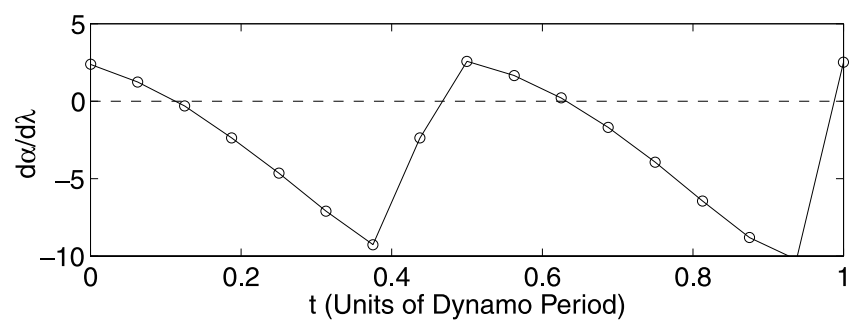

Figure 3. $d \alpha / d \lambda$ as a function of time covering the equivalent of two sunspot cycles. To find out the values of time which correspond to maxima or minima, look at Fig. 1 which has the same horizontal axis.

this effect, since the $\Sigma$-effect is cycle-independent (Longcope et al. 1998). Since it is in principle possible to determine from observational data how $d \alpha / d \lambda$ actually varies with the solar cycle, a plot like Fig. 3 provides a powerful tool for comparison between theory and observations. There is some indication in the existing data that $d \alpha / d \lambda$ may be varying in accordance with our model; but the data are noisy and the results from different instruments often diverge widely, so it is difficult to draw firm conclusions at this point in time (Pevtsov, private communication). 


\section{Conclusion}

Two clear theoretical predictions follow from our model.

(1) Since the helicity goes as $a^{-2}$ as seen from (3), the smaller sunspots should statistically have stronger helicity (i.e., higher values of twist $\alpha$ ).

(2) At the beginning of a cycle, helicity should be opposite of what is usually observed.

The alternative model for the generation of helicity, the $\Sigma$-effect proposed by Longcope et al. (1998), also makes the prediction (1), but not (2). Since helicity is imparted to the flux tubes by helical turbulence of SCZ in this model, the helicity is not expected to vary with the solar cycle. A careful analysis of any possible variation of helicity with the solar cycle would be the best way of ascertaining relative contributions of the $\Sigma$-effect and the dynamo (the process studied in this paper) in generating helicity.

\section{Acknowledgements}

Most of our calculations were carried out on the parallel cluster computer at Centre for High Energy Physics, Indian Institute of Science. P. C. acknowledges CSIR for financial support.

\section{References}

Abramenko, V. I., Wang, T., Yurchishin, V. B. 1997, Solar Phys., 174, 291.

Bao, S., Zhang, H. 1998, ApJ, 496, L43.

Canfield, R. C., Pevtsov, A. A. 2000, J. Astrophys. Astron., 21, 213.

Canfield, R. C., Hudson, H. S., McKenzie, D. E. 1999, Geophys. Res. Lett., 26(6), 627.

Chatterjee, P., Nandy, D., Choudhuri, A. R. 2004, A\&A, 427, 1019.

Choudhuri, A. R. 1998, The Physics of Fluids and Plasmas: An Introduction for Astrophysicists (Cambridge: Cambridge University Press).

Choudhuri, A. R. 2003, Solar Phys., 215, 31.

Longcope, D. W., Fisher, G. H., Pevtsov, A. A. 1998, ApJ, 507, 417.

Nandy, D., Choudhuri, A. R. 2002, Science, 296, 1671.

Nandy, D., Hahn, M., Canfield, R. C., Longcope, D. W. 2003, ApJ, 597, L73.

Pevtsov, A. A., Canfield, R. C., Metcalf, T. R. 1995, ApJ, 440, L109.

Pevtsov, A. A., Canfield, R. C., Latushko, S. M. 2001, ApJ, 549, L261.

Seehafer, N. 1990, Solar Phys., 125, 219. 